\title{
Stockage par échangeurs enterrés Influence de la zone non-saturée
}

\author{
Storage by buried heat exchangers \\ Influence of the unsaturated zone
}

\author{
J.P. Gaudet, S. Audibert, M. Menoud
}

Institut de Mécanique de Grenoble, LA n ${ }^{\circ} 6$

B.P. 68 - 38402 Saint Martin d'Hères

\begin{abstract}
On présente des résultats expérimentaux obtenus sur une réalisation de stockage intersaisonnier dans le sol de calories solaires à l'échelle d'un habitat unifamilial. Ces données ont été utilisées pour valider un modèle mathématique.

La résolution numérique du modèle a permis de tester l'influence de plusieurs paramètres (isolation, géométrie, niveau de température injectée...) sur le rendement $d u$ stockage.

Le risque d'assèchement du sol dù à la thermomigration est examiné, ainsi que la détermination et la signification des paramètres thermiques utilisés dans la modélisation.
\end{abstract}

This paper describes the experimental results obtained on interseasonal ground storage of solar heat at the scale of a single-family house. These data were used to validate a mathematical model.

The numerical resolution of the model was used to test the influence of several parameters (insulation, geometry, injected temperature level, etc.) on the efficiency of the storage.

The risk of drying out the soil by thermomigration is considered, as well as the determination and significance of the thermal parameters used in the model.

\section{Introduction}

Dans le cadre de l'utilisation rationnelle de l'énergie, une des voies explorées à l'Institut de mécanique de Grenoble concerne le stockage intersaisonnier de chaleur solaire dans la zone non-saturée du sous-sol. Ce type de stockage de chaleur sensible exploité par conduction thermique devait faire l'objet d'investigation, eu égard à la disponibilité du milieu de stockage (assurée presque partout) et à la facilité de mise en place d'un échangeur (faible profondeur).

L'étude correspond à un stockage de petite taille compatible avec le chauffage de quelques logements (nombre inférieur à 10). Une première phase a consisté à acquérir des résultats expérimentaux sur une réalisation de stockage intersaisonnier de calories solaires à l'échelle d'un habitat unifamilial. Les données ainsi obtenues ont été utilisées pour valider un modèle mathématique.

Le modèle, résolu numériquement, a permis de tester l'influence de plusieurs paramètres (isolation, géométrie,...) sur le fonctionnement du stockage. 


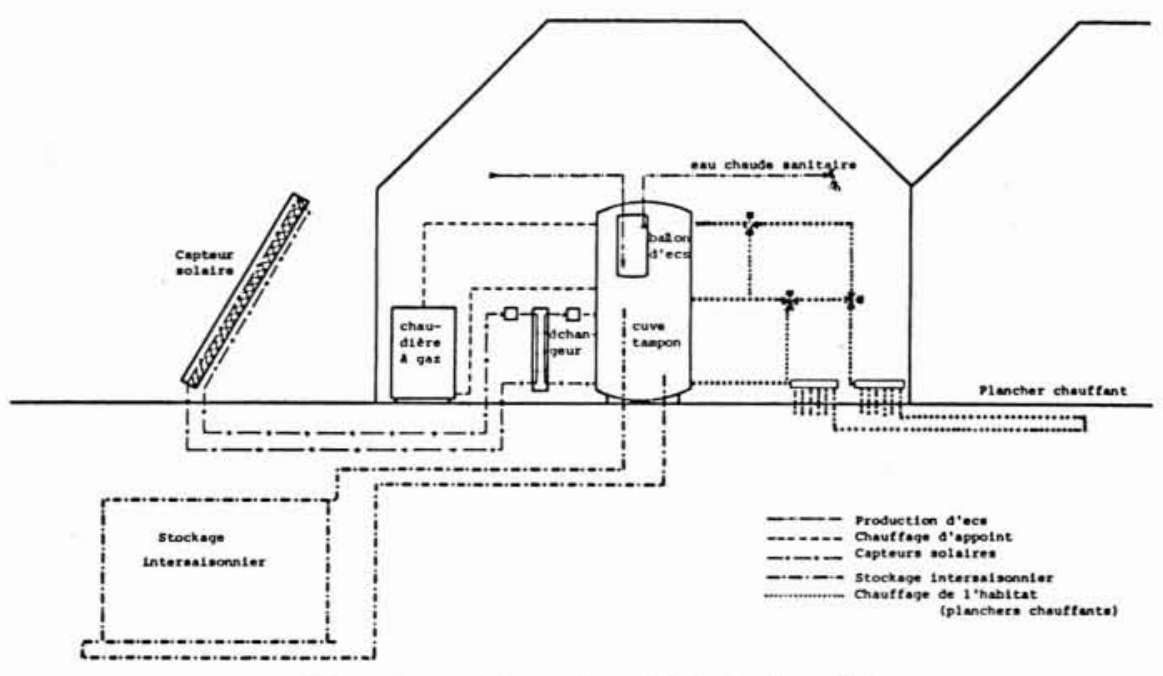

Figure 1. - Schéma de principe de l'installation.
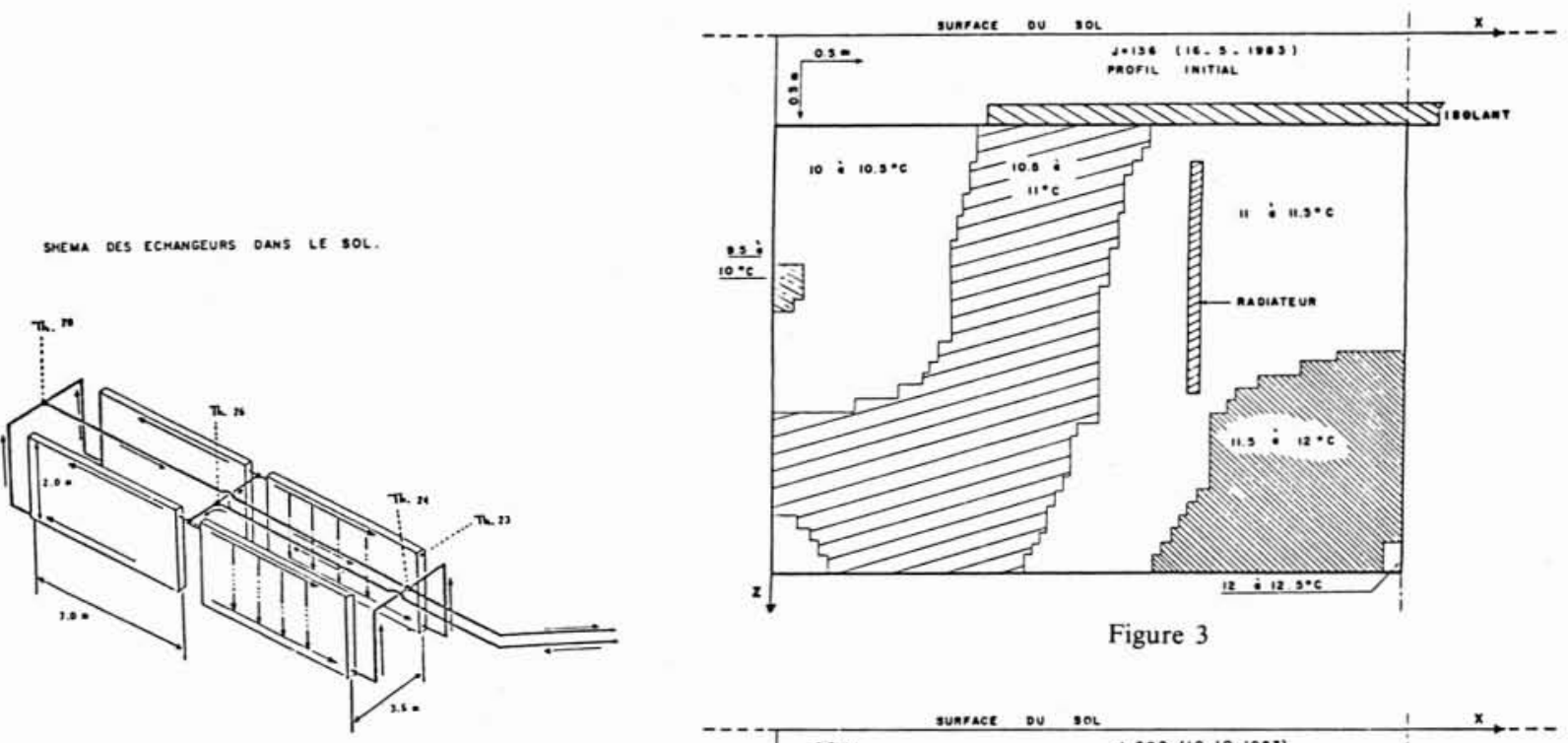

Figure 3
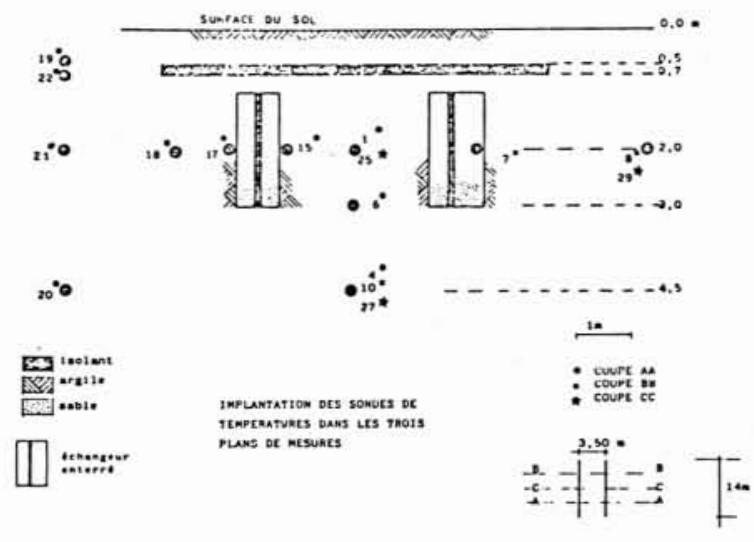

Figure 2

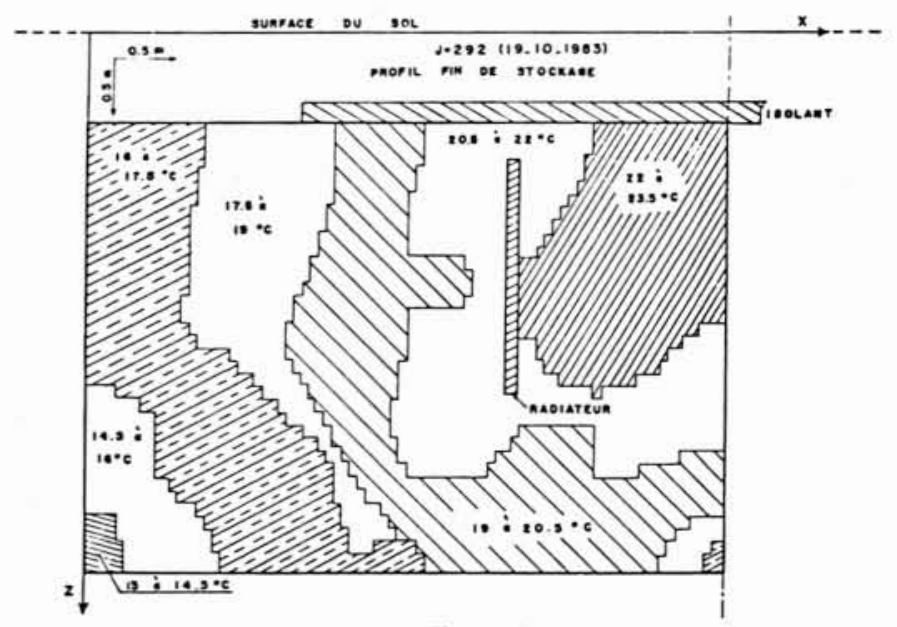

Figure 4 


\section{Installation expérimentale}

L'installation de chauffage solaire actif, avec stockage intersaisonnier, située à Meylan, (banlieue de Grenoble) est exploitée partiellement depuis mai 1982 et en totalité depuis octobre 1982.

L'ensemble est constitué de cinq boucles de fluide se fermant sur une même cuve tampon de $3 \mathrm{~m}^{2}$ d'eau ( $f$ gure 1), soit: des capteurs solaires (environ $50 \mathrm{~m}^{2}$ ), une chaudière d'appoint, une production d'eau chaude sanitaire, un stockage intersaisonnier dans le sol et des planchers chauffants.

Des sondes de température et des débitmètres permettent de connaitre les énergies et les températures mises en jeu dans chacune des boucles ainsi que l'énergie disponible dans la cuve tampon. Des mesures de température sont également faites dans la maison, à l'extérieur, dans le local technique et dans le sol contenant le stock intersaisonnier. Le rayonnement solaire global est mesuré dans le plan des capteurs.
Le stockage intersaisonnier comprend (figure 2):

- un échangeur enterré constitué de 2 rangées de radiateurs plans de chacune $2 \times 14 \mathrm{~m}$ distantes d'environ $3,5 \mathrm{~m}$;

- un milieu de stockage (chaleur sensible) qui est le sol du site;

- une isolation thermique limitant les pertes vers la surface du sol et formée d'une plaque horizontale de polyuréthane de dimensions $0,2 \times 7 \times 17 \mathrm{~m}$.

On peut estimer à $450 \mathrm{~m}^{3}$ le volume utile du stock.

La charge et la décharge thermique sont donc assurées par conduction dans le sol à partir de l'échangeur. Les quantités de chaleur chargées ou prélevées dans le sol transitent par la cuve tampon. La température de charge est conditionnée par la production d'eau chaude sanitaire. Le niveau thermique de déstockage est fixé par les planchers chauffants (basse température). Il est possible dans cette phase de mélanger l'énergie prélevée au stock et l'appoint de la chaudière.

\section{Résultats expérimentaux}

La charge thermique est faite manuellement en envoyant vers le stock intersaisonnier la chaleur stockée dans le bas de cuve. Il n'a pas été possible d'automatiser les charges, à cause d'une fuite hydraulique au niveau de l'échangeur enterré. La fuite n'ayant pas pu être localisée avec précision (et donc a fortiori réparée) le contrôle et le maintien de la pression de fonctionnement des autres boucles n'ont pu être assurés qu'avec des charges manuelles.

On présente, figures 3 et 4 , les champs thermiques mesurés au début et à la fin de la charge effectuée pendant l'été 1983.

Ces figures font ressortir deux points importants :

- l'état initial correspond sensiblement à un état naturel du sol, bien qu'une charge partielle ait été effectuée durant l'été précédent. Il semble donc qu'il n'y a aucun report de l'énergie non utilisée d'une année sur l'autre dans ce stock; - l'état final indique une température maximale de $23^{\circ} \mathrm{C}$ au cœur du stock pour une température moyenne de charge (imposée à l'entrée de l'échangeur enterré) de $49^{\circ} \mathrm{C}$.

La quantité totale d'énergie injectée dans cette charge est de $5100 \mathrm{kWh}$. Un calcul grossier à partir des résultats de ChOuard et al. (1977) donne pour cette charge une disponibilité solaire de $9000 \mathrm{kWh}$. Compte-tenu des pertes et de la consommation d'eau chaude sanitaire, la quantité, potentiellement chargeable est de $6700 \mathrm{kWh}$.

Malgré les charges journalières manuelles, $76 \%$ de l'énergie potentielle a été effectivement chargée.

On ne peut donc incriminer le mode de charge dans le comportement du stock.

Durant l'année 1983, les charges ont eu lieu jusqu'au 13 octobre. Le chauffage de l'habitat est assuré par les seuls capteurs solaires jusqu'au 15 novembre. A cette date, la température au cœur du stockage est d'environ $19^{\circ} \mathrm{C}$, alors que le niveau thermique minimal exploitable par les planchers chauffants est de $21^{\circ} \mathrm{C}$. Il est donc clair que, sur la saison $83-84$, le stock utilisé dans ces conditions n'a pu fournir aucune énergie en déstockage. Compte-tenu des résultats obtenus entre les saisons 82-83 et 83-84 (report très faible d'une année sur l'autre), on peut affirmer que sur cette installation le stockage intersaisonnier n'est pas possible.

L'analyse précise du fonctionnement du stock a été faite à partir d'un modèle mathématique. 


\section{Modèle mathématique}

Un modèle mathématique a été développé pour simuler les transferts thermiques dans le milieu de stockage. Ce modèle prend en compte :

- un transfert purement conductif dans le sol;

- un transfert convectif dans l'échangeur (radiateurs plans);

- des conditions aux limites de flux nul (en profondeur, à travers l'isolant, sur les plans de symétrie) ou de température variable et connue (à la surface du sol, à l'entrée de l'échangeur).

La résolution est faite numériquement par différences finies sur des mailles de grandes dimensions. Ce modèle, adapté à la simulation de longues périodes (plusieurs mois) restitue :
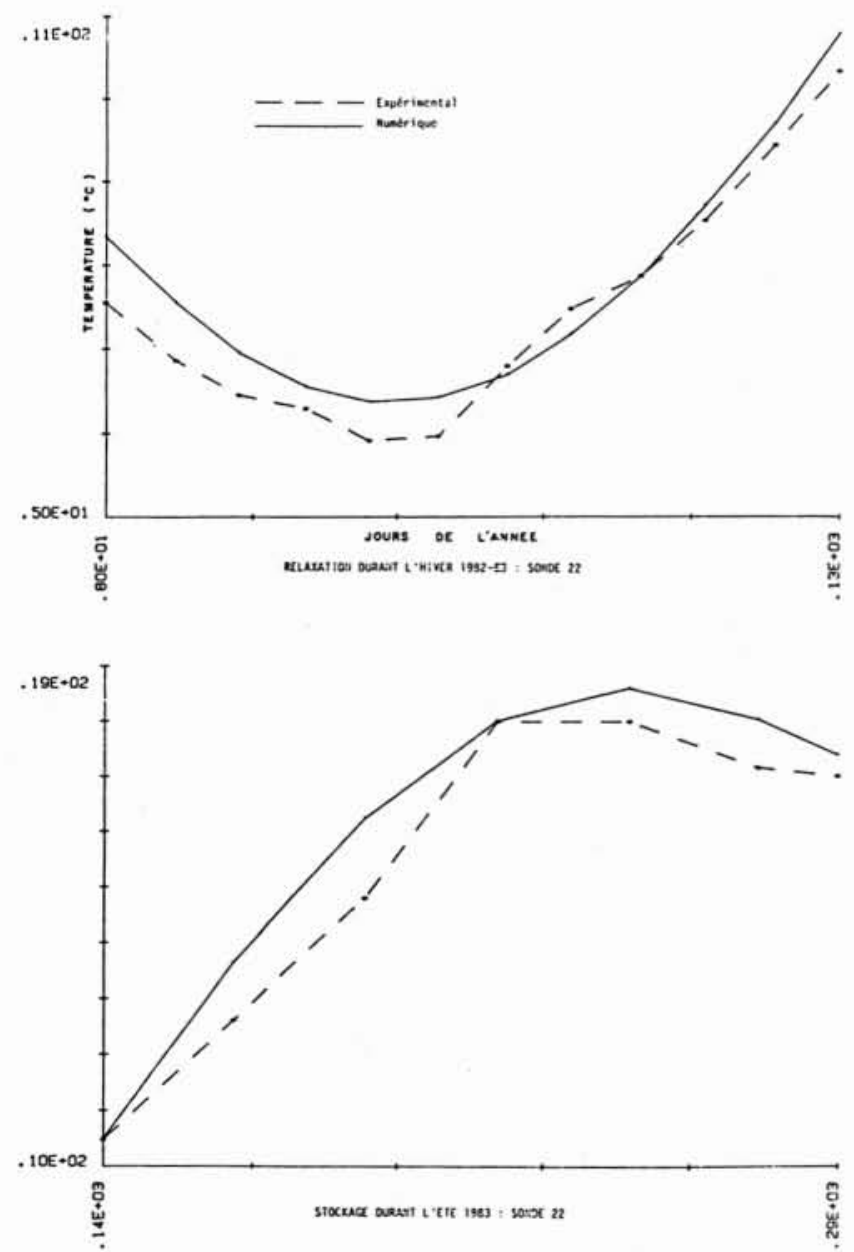

Figure 5
- l'évolution de la température de sortie de l'échangeur; - la quantité d'énergie stockée;

- les fuites thermiques vers la surface, horizontales et verticales à la limite du volume utile.

Le modèle validé sur les charges $82-83$ et $83-84$ a montré son adéquation à simuler les longues périodes. A titre d'exemple, on montre, figure 5, l'évolution des températures calculées et mesurées en un point du sol fortement influencé par la charge thermique et par la température de surface.

Les quantités d'énergie calculées et injectées expérimentalement sont également en bon accord.

Les paramètres thermiques (capacité calorifique et conductivité thermique) nécessaires au modèle ont été calés à partir d'essais préliminaires indépendants de celui présenté ici (PERSON, 1981).

Le sol constitutif du stock, très argileux, conserve toute l'année une teneur en eau très élevée. Ce résultat a été confirmé par des mesures tensiométriques de la pression de l'eau du sol (Person, 1981).

Ce comportement hydrique du sol est important pour différents aspects :

- l'hypothèse de constance temporelle des paramètres est vérifiée;

- un milieu poreux contenant une grande quantité d'eau liquide est plus résistant à l'asséchement près de l'échangeur (Person, 1981; Albergel, 1982).

L'asséchement du sol est néfaste au stockage car il provoque une diminution sensible de la capacité calorifique et modifie également la conductivité thermique apparente (AUDIBERT-GAUDET, 1984).

Le stockage de Meylan a été conçu pour limiter au maximum les effets de l'asséchement près de l'échangeur. Pour ce faire, la tranchée de mise en place des radiateurs a été remplie de sable saturé en eau. L'ensemble sableéchangeur a été enfermé dans une feuille plastique étanche en contact avec le sol en place. Les sols argileux présentent un retrait (gonflement) lors de cycles de séchage-humidification. Le sable a été choisi pour sa faculté à conserver ses propriétés mécaniques lors d'un éventuel séchage. On évite ainsi le retrait provoquant une résistance thermique de contact importante et limitant les échanges radiateur/sol.

Un autre point délicat de la modélisation concerne l'influence du stockage sur la température de surface du sol. La source de chaleur constituée par le stockage étant peu profonde, peut modifier la température naturelle de surface $T_{s}$. Il n'est alors plus possible d'utiliser des données météorologiques simples pour estimer $T_{s}$ et l'on doit prendre en compte le couplage. Pour nos conditions d'essais (isolant entre le stock et la surface) et compte-tenu des résultats obtenus par DUNAND 1982, Grima 1984 et BOUILLARD, 1984, il semble tout à fait correct de considérer la température de surface comme une donnée indépendante de la présence du stock. 


\section{Exploitation du modèle}

Le modèle développé et validé a été utilisé pour simuler d'autres conditions d'utilisation (température moyenne d'entrée, géométrie, surface d'isolant...).

On présente les résultats obtenus pour une même surface d'échangeur avec un stock plus compact ( 2 radiateurs plans de $5,5 \times 6,1 \mathrm{~m}$ distants de $3,5 \mathrm{~m}$ à la profondeur de $1 \mathrm{~m}$ ). La température moyenne d'injection est de $50^{\circ} \mathrm{C}$ pour une quantité totale d'énergie de $8780 \mathrm{kWh}$.

Trois configurations d'isolation de surface sont considérées : pas d'isolant, 1,75 et $3,5 \mathrm{~m}$ d'isolant en débordement des radiateurs.
Le tableau suivant regroupe les résultats obtenus sur 2 ans de simulation en considérant :

* le niveau énergétique atteint par la température au cœur du stock à la fin de la période de stockage (15 octobre);

* le report thermique d'une année sur l'autre par la température au cœur du stock à la fin de la période de chauffage de l'habitat (15 mai);

* le rendement énergétique atteint (quantité d'énergie récupérée sur énergie stockée en \%).

\begin{tabular}{|c|c|c|c|c|c|}
\hline & \multicolumn{3}{|c|}{ lère année } & \multicolumn{2}{|c|}{ 2ème année } \\
\hline & $\mid \begin{array}{c}\text { Temp. }\left({ }^{\circ} \mathrm{C}\right) \\
\text { au } 15 / 10\end{array}$ & $\begin{array}{l}\text { Temp. }\left({ }^{\circ} \mathrm{C}\right) \\
\text { au } 15 / 5\end{array}$ & $\begin{array}{l}\text { Rendement } \\
(\%)\end{array}$ & $\begin{array}{l}\text { Temp. }\left({ }^{\circ} \mathrm{C}\right) \\
\text { au } 15 / 10\end{array}$ & $\begin{array}{l}\text { Rendement } \\
(\%)\end{array}$ \\
\hline $\begin{array}{l}3,5 \mathrm{~m} \text { d'isolant au } \\
\text { delà des radiateurs }\end{array}$ & 41,6 & 21,4 & 13,2 & 45 & 20,5 \\
\hline $1,75 \mathrm{~m} d$ 'isolant & 41,5 & 19,0 & 12,1 & 44,3 & 17 \\
\hline pas d'isolant & 33 & 13,8 & 2,8 & 34,3 & 4,3 \\
\hline
\end{tabular}

\section{Conclusions}

Les résultats expérimentaux, complétés par la modélisation montrent que le stockage intersaisonnier de Meylan présente une surface développée trop importante par rapport à son volume. Les fuites thermiques, essentiellement vers la surface, sont trop importantes pour permettre le stockage jusqu'à la saison de chauffe de l'habitat.

La forme et la taille du stock ont été imposées lors de sa mise en place en 1980 par :

- les matériaux aisément disponibles pour l'échangeur (radiateurs plans);

- la mise en place (excavation d'une tranchée avec du matériel de génie civil).

D'autres stockages réalisés depuis utilisent des tuyaux verticaux plus faciles à mettre en place et beaucoup moins coûteux.
Des calculs complémentaires montrent qu'avec une même surface d'échangeur, mais une forme plus compacte et une isolation de surface importante, il est possible d'atteindre des rendements énergétiques de l'ordre de $20 \%$ à partir de la seconde année. Ce gain de rendement se fait au détriment du coût puisqu'il faut enfouir beaucoup plus profondément l'échangeur et rajouter environ $20 \%$ d'isolant de surface.

Dans les conditions favorables, la faible quantité d'énergie récupérée (environ $1780 \mathrm{kWh}$ la seconde année) alliée à un coût de mise en place élevé (excavation et isolant) semblent condamner le stockage intersaisonnier dans la zone non saturée pour des installations de petite taille. 


\section{Bibliographie}

Albergel A., 1982. - Analyse critique des modélisations des transferts de masse et de chaleur en milieu poreux non-saturé. Rapport de D.E.A., Université Scientifique et Médicale de Grenoble.

AUdibert S., GAUDET J.P., 1984. - Paramètres thermocinétiques apparents des milieux poreux et leur détermination par choc thermique. Comptes-rendus des Journées d'Étude du G.U.T. PARIS.

Bouillard D., 1984. - Étude d'un système d'extraction de calories du sol par pompe à chaleur. Expérimentation et modélisation analytique. Thèse de $3^{e}$ cycle, Université Scientifique et Médicale de Grenoble.
Chouard, Simon, Michel, 1977. - Bilan thermique d'une maison solaire. Méthode de calcul rapide. Rapport E.D.F.

DUNAND A., 1982. - Modélisation des transferts thermiques et hydriques dans le sol. Thèse de Docteur-Ingénieur, Université Scientifique et Médicale de Grenoble.

GrIMA M., 1984. - Modélisation des bilans thermiques des aquiféres à surface libre. Influence de la zone non-saturée et études des panaches de rejet de pompe à chaleur. Thèse de $3^{e}$ cycle, Université Scientifique et Médicale de Grenoble.

PERSON J.P., 1981. - Caractérisation des propriétés thermo-hydrauliques d'un sol non-saturé. Application au transport ou au stockage d'énergie par systèmes enterrés. Thèse de Docteur Ingénieur, Université Scientifique et Médicale de Grenoble.

\section{Discussion}

M. Ausseur. - Avec quelles fonctions de destockage les rendements présentés sont-ils obtenus?

M. GAUDET. - Les rendements calculés ont été obtenus à partir de deux fonctions de déstockage, une première qui exprime un apport complet par le stockage de l'énergie calorifique nécessaire, une seconde qui fait intervenir un chauffage d'appoint lorsque le niveau thermique du stockage devient insuffisant.

\section{tion?}

M. SourieaU. - Avez-vous calculé le coût d'une telle opéra-

M. GAUDET. - C'est une étude à caractère de recherche. Elle n'a pas été optimisée du point de vue économique. Il faudrait utiliser des tubes verticaux. On avait été obligé d'installer une protection cathodique pour éviter la corrosion. Le surcoût est énorme, le coût de l'énergie stockée approchant celui des batteries au plomb.

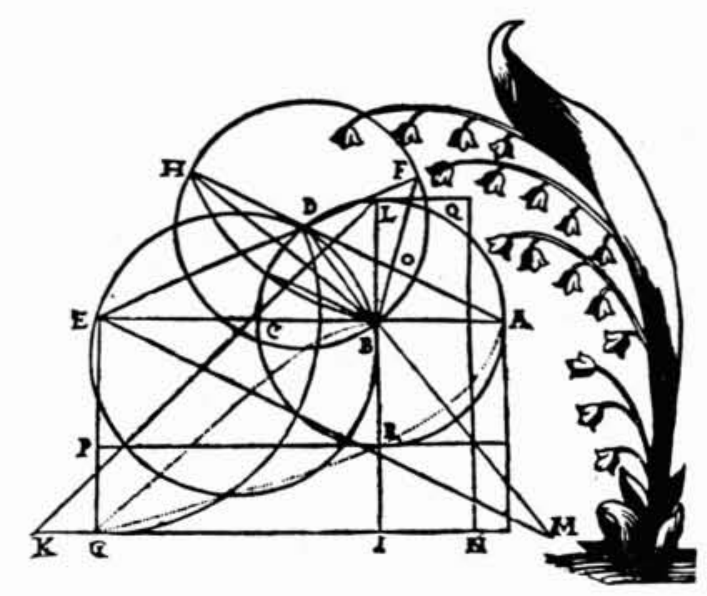

